\title{
UNIQUENESS OF RADIAL SOLUTIONS OF SEMILINEAR ELLIPTIC EQUATIONS
}

\author{
MAN KAM KWONG AND YI LI
}

\begin{abstract}
E. Yanagida recently proved that the classical Matukuma equation with a given exponent has only one finite mass solution. We show how similar ideas can be exploited to obtain uniqueness results for other classes of equations as well as Matukuma equations with more general coefficients. One particular example covered is $\Delta u+u^{p} \pm u=0$, with $p>1$. The key ingredients of the method are energy functions and suitable transformations. We also study general boundary conditions, using an extension of a recent result by Bandle and Kwong. Yanagida's proof does not extend to solutions of Matukuma's equation satisfying other boundary conditions. We treat these with a completely different method of Kwong and Zhang.
\end{abstract}

\section{INTRODUCTION}

In a recent preprint [24], E. Yanagida established the uniqueness of the finite mass solution of the equation

$$
\Delta u(x)+\frac{u^{p}(x)}{1+|x|^{2}}=0, \quad x \in \mathbb{R}^{n},
$$

where $n \geq 3$ and $1<p<(n+2) /(n-2)$. This equation was first proposed by Matukuma [18] as a mathematical model of a globular cluster of stars. The question of existence and symmetry properties of ground states has been studied extensively by $\mathrm{Li}$ and $\mathrm{Ni}[16,17], \mathrm{Ni}$ and Yotsutani [21], and Noussair and Swanson [22]. Yanagida's uniqueness proof made some innovative use of the Pohozaev identity. The complexity of the identity, however, has not helped to elucidate the ingenious underlying arguments. We find that similar techniques are applicable to a wider class of problems. We give several examples in this paper.

In $\S 2$ we consider the Dirichlet problem for equations of the form

$$
u^{\prime \prime}(x)+f(u(x))+g(x) u(x)=0, \quad x \in(a, b) .
$$

Received by the editors June 11, 1990.

1991 Mathematics Subject Classification. Primary 34B15; Secondary 35J25, $35 \mathrm{~J} 65$.

Key words and phrases. Semilinear elliptic equation, boundary value problem, uniqueness, radial solution, positive solution, energy function.

The work of the first author was supported by the Applied Mathematical Sciences subprogram of the Office of Energy Research, U. S. Department of Energy, under Contract W-31-109-Eng-38.

The work of the second author was supported by NSF Grant NSF-DMS 8603627. 
The coefficient $g(x)$ is assumed to be $\wedge$-shaped. The energy function for this equation is particularly simple, and the proof serves to illustrate the main ideas without additional computational technicalities.

In $\S 3$ we use the same method together with the classical Kelvin transformation (made famous by Fowler in his extensive study of the Emden-Fowler equation) to study equations of the form $\Delta u+u^{p}+q(|x|) u=0$. Specializations to constant $q(|x|)$ give a simpler alternative proof of the result in [15] as well as an affirmative answer to the open conjecture concerning the uniqueness of the positive solution of

$$
\Delta u+u^{p}+u=0, \quad u(x)=0 \quad \text { on } \partial B_{b},
$$

where the exponent $p \in(1,(n+2) /(n-2))$ is subcritical and $\partial B_{b}$ is the sphere of radius $b$ in $\mathbb{R}^{n}$. This equation has been studied by Brezis and Nirenberg in their well-known paper [3]. L. Zhang has recently [25] informed us that he also had solved the conjecture using a different method. Our result allows a coefficient in the linear term and covers Dirichlet problems on annular regions.

In $\S 4$ we first extend a result in Bandle and Kwong [2] to enable us to apply the previous results to boundary value problems with more general homogeneous conditions. We also give other results for situations where the extended BandleKwong theorem may not work.

In $\S 5$ we indicate how Yanagida's proof also works for more general coefficients in the Matukuma equation. The Kelvin transformation again helps to clarify the arguments.

In $\S 6$ we consider other boundary value problems on the generalized Matukuma's equation, with a general exponent that need not be subcritical. Yanagida's method is tied to the asymptotic behavior of the ground state and therefore is no longer applicable. We establish uniqueness under a stronger condition on the coefficient. The method used is that of Kwong and Zhang [15]. Further extensions of the method are also indicated.

In $\S 7$ we establish a uniqueness result on fixed-end boundary value problems and give some examples that are not covered by the results in the previous sections but can be treated by using extensions of the arguments presented here and in previous work. Some of our assertions are not formulated in the most general form possible, but only with respect to the examples.

Acknowledgment. Most of the complicated analytic computations needed for this research have been performed with the help of the symbolic algebra software MAPLE. These include the various transformations on the equations and the verification of the uniqueness criteria.

\section{THE EMDEN-FOWLER EQUATION-DIRICHLET PROBLEM}

In this section, we prove a uniqueness result for boundary value problems of the form

$$
\begin{gathered}
u^{\prime \prime}(x)+f(u(x))+g(x) u(x)=0, \quad u(x)>0, \quad x \in(a, b), \\
u(a)=0, \quad u(b)=0 .
\end{gathered}
$$

Here $(a, b)$ is a bounded interval, and $f(u)$, and $g(x)$ are continuous functions. To ensure that solutions of initial value problems are unique and depend 
continuously on the initial conditions, we require that $f(u)$ be locally Lipschitz continuous. Denote by $F(u)$ the integral of $f(u)$ :

$$
F(u)=\int_{0}^{u} f(s) d s .
$$

We assume that $f(u)$ is superlinear, in other words,

$$
f(u) / u>0 \text { is a strictly increasing function of } u \text {. }
$$

The condition we shall impose on $g(r)$ is

$$
\begin{aligned}
& \text { there is a point } c \in[a, b] \text { such that } g(x) \text { is } \\
& \text { nondecreasing in }[a, c] \text { and nonincreasing in }[c, b] \text {. }
\end{aligned}
$$

For convenience, we call condition (2.5) the $\wedge$-property of $g(x)$. Note that (2.5) includes the special cases when $g(x)$ is nondecreasing or nonincreasing in the entire interval $[a, b]$ (corresponding to $c=b$ or $c=a$, respectively). It is natural to ask whether a $\vee$-property on $g(x)$ can work just as well to give uniqueness. We shall give a counterexample in $\S 3$. On the other hand, in $\S 7$ we shall see that uniqueness may still hold for many $\vee$-shaped $g(x)$.

Note that even though $f(u)=u^{p}$ does not satisfy $(2.4)$, with $0<p<1$ (and more generally for sublinear equations such that $f(u) / u$ is a nonincreasing function of $u$ ), uniqueness has been shown to prevail. Therefore (2.4) is not a necessary condition. All powers of $u$ with exponents larger than unity satisfy (2.4), as do sums of positive multiples of powers. Strict monotonicity on the functions involved is imposed to rule out the linear case, for which uniqueness is certainly not valid. There is obviously room for weakening the strictness requirement, but to simplify the presentation, we shall not pursue this issue. The following property of superlinearity is well known and routinely applied. We outline a brief proof for the sake of easy reference.

Lemma 1. Let $u(x)$ and $U(x)$ be two distinct solutions of $(2.1)$. If $u(x) \leq$ $U(x)$ in some subinterval $[c, d]$ of $(a, b)$ and $u^{\prime}(c) / u(c) \geq U^{\prime}(c) / U(c)$, then

$$
u^{\prime}(d) / u(d)>U^{\prime}(d) / U(d) \text {. }
$$

Furthermore, if $u(x)$ vanishes at some point $\tau>d$, then $U(x)$ must intersect $u(x)$ in $(d, \tau)$. Likewise, if $u^{\prime}(c) / u(c) \leq U^{\prime}(c) / U(c)$ and $u(x)$ vanishes at some point $\sigma<c$, then $U(x)$ must intersect $u(x)$ in $(\sigma, c)$.

Proof. By rewriting the respective differential equations in the "linear" forms $u^{\prime \prime}+[f(u) / u+g(x)] u=0$ and $U^{\prime \prime}+[f(U) / U+g(x)] U=0$, we see that the second has a larger coefficient for the linear term. Hence, according to the oscillation theory of linear differential equations, $u(x)$ oscillates more slowly than $U(x)$ in $[c, d]$. The conclusions are then simple consequences of Sturm's comparison theorem.

The rest of this section is devoted to proving the following theorem. Extensions to more general boundary value problems and to equations obtained by replacing the linear term in (2.1) with other powers $u^{q}$ are given in $\S 4$.

Theorem 1. Under the assumptions (2.4) and (2.5), the boundary value problem (2.1)-(2.2) has at most one positive solution. 
The $\wedge$-condition on $g(x)$ appears at first to be similar to that in the wellknown Moroney uniqueness criterion (see Kwong [11, 14] for improved versions). However, what makes (2.5) interesting is that there is no restriction on the location of the point $c$. Even in the particular cases when $c=a$ or $b$, the result is unexpected because for general superlinear equations, monotonicity alone of the coefficient $g(x)$ is not sufficient for the uniqueness of the Dirichlet problem. The Moroney condition works only for Neumann-Dirichlet problems, and extension to the full Dirichlet problem requires additional monotonicity requirements on $g(x)$.

Suppose there are two distinct solutions, $u(x)$ and $U(x)$, to the boundary value problem. Let us derive a contradiction. First we observe that $u(x)$ and $U(x)$ must intersect somewhere inside $(a, b)$. Suppose instead that $u(x)<$ $U(x)$ in $(a, b)$. Then by Lemma $1, U(x)$ oscillates faster than $u(x)$; and hence by Sturm's separation theorem, $U(x)$ must have a zero within the two consecutive zeros $a$ and $b$ of $u(x)$. This contradicts the fact that $U(x)>0$ inside $(a, b)$.

Let us next dispose of the case in which $u(x)$ and $U(x)$ intersect exactly once at $\sigma \in(a, b)$. Suppose the solutions have been named such that

$$
u^{\prime}(a)<U^{\prime}(a) \text {. }
$$

Then we have $u(x)<U(x)$ for $x \in(a, \sigma)$ and $u(x)>U(x)$ for $x \in(\sigma, b)$. Define $r(x)=u^{\prime}(x) / u(x)$ and $R(x)=U^{\prime}(x) / U(x)$. We claim that $r(x) \neq$ $R(x)$ in $(a, b)$. Suppose the contrary, that there exists a point $\rho$ such that $r(\rho)=R(\rho)$. If $\rho>\sigma$, we see from Lemma 1 that $U(x)$ must bend down to intersect $u(x)$ at least one more time in $(\sigma, b)$. This contradicts the assumption that $\sigma$ is the only intersection point. Likewise, if $\rho<\sigma$, Lemma 1 gives the contradiction that $U(x)$ must meet $u(x)$ again in $(a, \sigma)$.

Since $r(\sigma)>R(\sigma)$, we have $r(x)>R(x)$ for all $x \in(a, b)$. It follows upon integrating the inequality that the ratio $u(x) / U(x)$ is a strictly increasing function of $x$ in $(a, b)$. Define

$$
\gamma=u(c) / U(c)
$$

Then

$$
u(x) / U(x)<\gamma \text { for } x<c,
$$

and

$$
u(x) / U(x)>\gamma \text { for } x>c .
$$

Let us study the energy function

$$
E(u(x))=u^{\prime 2}(x)+2 F(u(x))+g(x) u^{2}(x),
$$

defined for any given solution $u(x)$. Differentiating (2.11) gives

$$
E^{\prime}=g^{\prime}(x) u^{2}
$$

and so

$$
u^{\prime 2}(b)=u^{\prime 2}(a)+\int_{a}^{b} g^{\prime}(x) u^{2}(x) d x
$$


For simplicity we have assumed that $g(x)$ is differentiable. The proof still works in the general case by interpreting integrals such as the one in (2.13) in the Stieljes sense.

Applying the same computation to $U(x)$ and multiplying the resulting identity by $\gamma^{2}$, we obtain

$$
\gamma^{2} U^{\prime 2}(b)=\gamma^{2} U^{\prime 2}(a)+\int_{a}^{b} g^{\prime}(x) \gamma^{2} U^{2}(x) d x .
$$

Let us compare the various terms in (2.13) and (2.14). A contradiction is eventually derived by first showing that the left-hand side of $(2.13)$ is not less than that of (2.14), and then showing that the right-hand side of $(2.13)$ is not larger than that of (2.14), with strict inequality in at least one of the cases.

Taking the limit of (2.9) with $x \rightarrow b$ and using L'Hospital's rule, we see that

$$
u^{\prime 2}(b) \geq \gamma^{2} U^{\prime 2}(b) \text {. }
$$

Note that equality holds if $c=b$. The same arguments apply to the other endpoint $a$, except that the direction of the inequality is reversed:

$$
u^{\prime 2}(a) \leq \gamma^{2} U^{\prime 2}(a)
$$

Also note that strict inequality must be true in at least one of (2.15) and (2.16). Finally, the integral in (2.14) is smaller than that in (2.13) because, by (2.5), (2.9), and (2.10), the integrand in the following integral is always negative:

$$
\int_{a}^{b} g^{\prime}(x)\left(u^{2}(x)-\gamma^{2} U^{2}(x)\right) d x
$$

This completes the proof for the case of a single intersection point.

Now suppose that $u(x)$ and $U(x)$ intersect more than once in $(a, b)$. We use a continuous deformation argument to reduce this to the former case. This technique will be used again in the proof of Theorem 4 in $\S 4$. We assume that $u^{\prime}(a)<U^{\prime}(a)$. We use a shooting argument. We let $u(x ; \alpha)$ be the solution of (2.1) satisfying the initial conditions

$$
u(a ; \alpha)=0 \text { and } u^{\prime}(a ; \alpha)=\alpha .
$$

The original $u(x)$ is then imbedded as a member of this family of solutions, namely, $u(x)=u\left(x, u^{\prime}(a)\right)$. As $\alpha$ is altered, $u(x ; \alpha)$ varies continuously. We start with $\alpha$ equal to the initial slope of the given solution $u(x)$ and decrease it progressively. Let us track the position of the second intersection point of $u(x, \alpha)$ and $U(x)$. By assumption, initially this point is in the interior of $(a, b)$. As $\alpha$ varies, the point moves continuously along the curve of $U(x)$. It cannot reach the left endpoint $a$ because (as we shall show in a moment) two intersection points cannot occur too close together. If it reaches $b$ for some $\bar{\alpha}$, then we have two distinct solutions $U(x)$ and $u(x ; \bar{\alpha})$ of the Dirichlet problem that intersect exactly once in $(a, b)$, a case already shown to be impossible. We have implicity used the fact that the part of $u(x, \alpha)$ between $a$ and the first intersection point can never touch the $x$-axis, a consequence of the uniqueness result for initial value problems.

Hence we conclude the second intersection point is in the interior of the interval $(a, b)$ no matter what $\alpha$ is. By choosing $\alpha>0$ sufficiently small, we can make the first intersection point of $u(x ; \alpha)$ and $U(x)$ arbitrarily close 
to $b$. The distance between the first and second intersection points is then arbitrarily small. This means that the function $v(x)=u(x ; \alpha)-U(x)$ oscillates very fast within a short distance. This function satisfies the differential equation

$$
v^{\prime \prime}(x)+\left[\frac{f(u(x))-f(U(x))}{u(x)-U(x)}+g(x)\right] v(x)=0 .
$$

By the Lipschitz continuity of $f(u)$, the fraction in the coefficient of $v(x)$ is bounded; hence the entire coefficient is bounded. A simple Sturm comparison argument (or an application of the well-known Lyapunov inequality) shows that oscillation within an arbitrarily short interval is impossible. We thus have a contradiction, and the proof of the theorem is complete.

$$
\text { 3. The equation } \Delta u+u^{p}+q(r) u=0
$$

The study of the uniqueness of the ground state of the equation

$$
\Delta u+u^{p}-u=0 \text { in } \mathbb{R}^{n},
$$

as well as solutions of the Dirichlet problem on any finite ball, started with the paper of Coffman [6], was carried on by McLeod and Serrin [19], and was completed by Kwong [12]; refer to [12 and 15] for more details. More recent work on equations with a general nonlinear term has been done by Chen and Lin [5], who also made use of the Phozaev inequality.

In [3], Brezis and Nirenberg studied the nonlinear eigenvalue problem on the unit ball $B$ :

$$
\Delta u+u^{p^{*}}+\lambda u^{q}=0 \text { in } B, \quad u>0 \text { in } B,
$$

with the Dirichlet boundary condition

$$
u=0 \text { on } \partial B \text {, }
$$

where $1<q<p^{*}=(n+2) /(n-2)$. Using variational techniques, they obtained necessary and sufficient conditions on the value of $\lambda$ for the existence of a solution. What is most interesting is that the necessary and sufficient range depends on the value of $q$ as well as on $n$. Two or three cases can be distinguished according to whether $n \geq 4$ or $n=3$. For all values of $n$, the cutoff value of $q$ for the first case is $n /(n-2)$, whereas for $n=3$, the value $q=1$ is in a category by itself. Atkinson and Peletier [1] made a further study of the equation using the shooting method. They showed that when $q>1$, uniqueness is generally not true.

Here we are concerned only with the case where the lower-order term $u^{q}$ is linear. The pertinent existence result in [3] follows.

Theorem (Brezis and Nirenberg). Let $q=1$ in equation (3.2). For $n=3$, the boundary value problem (3.2)-(3.3) has solution if and only if $\lambda \in\left(\pi^{2} / 4, \pi^{2}\right)$. For $n \geq 4$, the same boundary value problem has a solution if and only if $\lambda \in\left(0, \lambda_{1}\right)$, where $\lambda_{1}$ is the first eigenvalue of the Laplacian $-\Delta$ with the Dirichlet boundary condition (3.3).

A question left open was whether the solution guaranteed by the theorem is unique. Numerical evidence points to an affirmative answer.

In this section we use the techniques presented in $\S 2$ to derive a general result covering both of these cases, providing a simpler alternative proof of the results 
in [12] and an affirmative answer to the Brezis and Nirenberg conjecture. Our method allows us to include a coefficient $q(r)$ in the linear term. In the next section we extend our results to boundary value problems in an annular region with more general boundary conditions.

We study the following equation on a ball of radius $b$ :

$$
\Delta u+u^{p}+q(r) u=0, \quad u>0 \text { in } B_{b}, p>1,
$$

with a radially symmetric coefficient $q(r), r=|x|$, and subject to the boundary condition

$$
u=0 \quad \text { on } \partial B_{b} \text {. }
$$

The condition we impose on $q(r)$ is

$$
G(r):=\left[r^{\beta} q(r)-L r^{\beta-2}\right] \text { has the } \wedge \text {-property, }
$$

where $\beta$ and $L$ are constants given by

$$
\beta=\frac{2 m(p-1)}{p+3}, \quad L=\frac{2 m(m p+m-p-3)}{(p+3)^{2}} \quad(m=n-1) .
$$

Direct computation will verify that when $q(r)$ is a negative constant, then (3.6) is satisfied for all $p>1$, whereas when $q(r)$ is a positive constant, (3.6) is satisfied only for subcritical exponents, $1<p<(n+2) /(n-2)$. Although for the sake of simplicity Theorem 2 is stated only for a finite ball, it is not difficult to see that the same result holds for ground states (solutions defined in the entire $\mathbb{R}^{n}$ such that $\left.\lim _{r \rightarrow \infty} u(r)=0\right)$. Hence our result here applies to both (3.1) and (3.2). It is known from a result of Ni and Nussbaum [20] (see also Budd and Norbury [4]) that in the remaining case of a positive constant $q(t)$ and supercritical $p$, uniqueness is no longer valid. The expression in (3.6) satisfies the $V$-property instead. This furnishes the counterexample promised in the last section.

Theorem 2. Let $n \geq 3$. Under the hypothesis (3.6), the problem (3.4)-(3.5) has at most one radially symmetric solution.

From a well-known theorem of Gidas, Ni, and Nirenberg [9, 10], we know that when $q(r)$ is a constant or a nonnegative nonincreasing function, all solutions of the boundary value problem must be radially symmetric; then we obtain absolute uniqueness. The interesting recent work of Dancer [8] illustrates how the lack of symmetry in the spatial domain affects uniqueness.

Proof. For radially symmetric solutions, (3.4) reduces to an ordinary differential equation

$$
u^{\prime \prime}(r)+\frac{m}{r} u^{\prime}(r)+u^{p}+q(r) u=0,
$$

where $m=n-1$, with boundary conditions

$$
u^{\prime}(0)=0, \quad u(b)=0 .
$$

We apply the classical transformation

$$
u(r)=v(r) / r^{\alpha},
$$

with

$$
\alpha=2 m /(p+3),
$$


to obtain

$$
r^{\beta} v^{\prime \prime}+K r^{\beta-1} v^{\prime}+v^{p}+\left[r^{\beta} q(r)-L r^{\beta-2}\right] v=0
$$

where

$$
K=\frac{m(p-1)}{p+3},
$$

and $\beta$ and $L$ are given by (3.7). The equation looks complicated, but each coefficient is just a multiple (either by a constant or by $q(r)$ ) of some power of $r$. The constant $\alpha$ in (3.10) has been chosen as in (3.11) in order that, in (3.12), the derivative of the coefficient of $v^{\prime \prime}$ is twice the coefficient of $v^{\prime}$. This makes the derivative of the following energy function particularly simple:

$$
E(v)=t^{\beta} v^{\prime 2}+\frac{2 v^{p+1}}{p+1}+G(r) v^{2},
$$

where $G(r)$ is the expression inside the square brackets in (3.12). More precisely,

$$
\frac{d E(v)}{d r}=G^{\prime}(r) v^{2}
$$

It is now clear that we are in a completely analogous situation to that of the last section, and the same arguments apply to give the required uniqueness conclusion. One technical point we must address is that $G(r)$ can tend to $-\infty$ as $r \rightarrow 0$, if the exponent of $r$ in the second term is negative. This, however, does not pose any real difficulty because the term is multiplied by $v^{2}(r)$ which tends to 0 fast enough to cancel out the singularity; indeed we have $\lim _{r \rightarrow 0} G(r) v^{2}(r)=0$. We omit the detailed verification.

Corollary 1. If $p$ is subcritical and $r^{\beta} q(r)$ is nondecreasing, then (3.4)-(3.5) has a unique solution.

Proof. By assumption, the first term, $r^{\beta} q(r)$, in $G(r)$ is increasing. That the constant $L$ is positive follows from the assumptions that $m \geq 2$ and $p>1$; so the second term in $G(r)$ is negative. Using the fact that $p$ is subcritical, one can easily see that the exponent in $r^{\beta-2}$ is negative. As a result, the second term in $G(r)$ is also increasing. Hence (3.6) is satisfied.

Note that if we convert $v(r)$ back to $u(r)$, the (3.14) and (3.15) become the familiar Pohozaev identity. Hence the use of the Kelvin transformation is in essence equivalent to the use of Pohozaev's identity. The treatment of a much simpler situation in the foregoing section brought out the desirable characteristic of an energy function that makes the proof successful-the derivative of the energy is a suitable multiple of the square of the solution function. This led us to look for a desirable form of the differential equation with such an energy function. We came up with equations of the form (3.12), which we recognized to be one obtainable from (3.4).

It is worthwhile to evaluate and compare the approach of this paper and the Coffman-Kolodner approach used in [12]. At first sight, the new method appears to be far superior to the old one. It is much simpler and is applicable to equations having a coefficient $q(r)$. Later we shall also see that the new proof works without change for the Dirichlet problem, while that in [12] is hopelessly 
bonded to the Neumann-Dirichlet problem. However, the recent work of Kwong and Zhang [15] and Chen and Lin [5] demonstrated that the Coffman-Kolodner method can be used to deal with $\Delta u+f(u)=0$ for a wide class of nonlinear $f(u)$. A simple example is $f(u)=u^{p}-u^{q}$, with $p>q>1$. We attempted but failed to modify the new method to treat $q>1$. Furthermore, the result of Kwong and Zhang is the only known one that covers functions that are neither superlinear nor sublinear. In $\S 6$ we give a further application of the CoffmanKolodner approach in the study of the generalized Matukuma equation, for which the new method is utterly inadequate. In conclusion, each of the two methods has its own merits and drawbacks. Both deserve to be kept inside our toolbox.

By choosing an appropriate $\alpha$ in (3.11), we can also reduce equations of the form

$$
u^{\prime \prime}(r)+\frac{m}{r} u^{\prime}(r)+r^{\gamma} u^{p}+q(r) u=0
$$

to one amenable with our technique. More generally, equations of the form

$$
u^{\prime \prime}+A(r) u^{\prime}+B(r) u^{p}+C(r) u=0
$$

can be first transformed to

$$
u^{\prime \prime}+\rho(r) u^{p}+q(r) u=0
$$

by a change of the independent variable. Next, the change of the dependent variable, $u(r)=[\rho(r)]^{-1 /(p+3)} v(r)$, transforms the equation into a new one having an energy function of the type required in our proof. We state below one uniqueness criterion obtained by this method for equations with $q(r)=0$, and skip the less elegant result for the most general situation.

Theorem 3. Suppose that

$$
(p+3)^{2} \rho^{2} \rho^{\prime \prime \prime}-3(p+3)(p+5) \rho \rho^{\prime} \rho^{\prime \prime}+2(p+4)(p+5)\left[p^{\prime}\right]^{3}
$$

is negative in $(a, c)$ and positive in $(c, b)$ for some $c \in[a, b]$. Then the differential equation

$$
u^{\prime \prime}(r)+\rho(\gamma) u^{p}(r)=0
$$

has at most one nonnegative solution satisfying the Robin boundary conditions (4.1) and (4.2).

A test class of coefficients that can be used to try out this criterion is

$$
\rho(r)=\lambda r^{\alpha}+\mu r^{\beta}, \quad-\infty<\lambda, \mu, \alpha, \beta<\infty .
$$

We can take $\lambda=1$ since the criterion is invariant under constant multiples. We can also assume $\mu=1$ using a (horizontal) scaling argument. The actual computation is very involved. We have investigated the case $\beta=0$, with the help of the algebraic manipulation software MAPLE. With $\rho=r^{\alpha}+1$ the expression in (3.19) turns out to be $\alpha r^{2}$ times

$$
\begin{aligned}
k(R)= & 2(p+2 \alpha+3)(p+\alpha+3) R^{2} \\
& -(\alpha-1)(p+3)(p \alpha+4 p+9 \alpha+12) R \\
& +(\alpha-1)(\alpha-2)(p+3)^{2}
\end{aligned}
$$


where $R=r^{2}$. The uniqueness condition of Theorem 3 requires that $k(R)$ has at most one positive root and the coefficient of $R^{2}$ is positive. For $1 \leq \alpha \leq 2$, the last term of $k(R)$ is negative and so the criterion is satisfied. For $\alpha<1$, the uniqueness criterion is satisfied only if $k(R)$ does not have two distinct real roots. We computed the discriminant and obtained the nicely factored form

$$
\Delta=(p+3)^{2}(p+5) \alpha^{2}(\alpha-1)(p \alpha+13 \alpha+7 p+19),
$$

which is nonpositive if $\alpha \geq-(7 p+19) /(p+13)$. We have thus established uniqueness for the range

$$
-\frac{7 p+19}{p+13} \leq \alpha \leq 2
$$

There is no reason to believe that this range is optimal.

Related but independent results for the same class of coefficients have been obtained by Kwong in [14]. The uniqueness range $-2 \leq \alpha \leq 2$ has already been established.

Two other interesting examples not included in the results of [14] are

$$
\rho(r)=\left(1+r^{2}\right)^{2}
$$

and

$$
\rho(r)=r+\frac{1}{r}
$$

In the latter case, the expression in (3.19), after multiplying by $r^{6} / 2$ and substituting $R$ for $r^{2}$, is

$$
\begin{aligned}
k(R)= & \left(p^{2}+9 p+20\right) R^{3}-\left(9 p^{2}+69 p+132\right) R^{2} \\
& -\left(3 p^{2}+9 p-6\right) R-\left(p^{2}+3 p+2\right) .
\end{aligned}
$$

It is required to show that $k(R)$ has only one positive root (for all $p$ ). At $R=0, k(0)<0$. Therefore, $k(R)$ has either one or three (counting multiple roots) positive roots. On the other hand, $k^{\prime}(0)<0$. A simple picture is enough to convince one that the existence of three positive roots will imply the existence of three positive critical points, contradiction the fact that $k(R)$ is only cubic.

\section{General homogeneOUS BOUNDARY CONDITIONS}

We wish to extend the results obtained in the previous two sections to cover more general boundary conditions, those of the homogeneous and separated type:

$$
u^{\prime}(a)+A u(a)=0
$$

and

$$
u^{\prime}(b)+B u(b)=0 .
$$

Boundary conditions of the type (4.1) or (4.2) has been called the Robin type. The cases $A=\infty$ and $B=\infty$ are taken to mean the usual Dirichlet boundary conditions, $u(a)=0$ and $u(b)=0$, respectively. 
To this end, we first obtain an extension of a result by Bandle and Kwong [2], who confirmed a conjecture raised and partially resolved by Coffman and Marcus in [7] concerning the universal uniqueness property of Dirichlet boundary value problems for superlinear equations:

Suppose it is known that given any bounded interval $(a, b)$, the Dirichlet problem has a unique positive solution. It is also known that every solution of a given Neumann-Dirichlet problem can be extended to the left until the solution crosses the $r$-axis. Then the Neumann-Dirichlet problem has also a unique solution.

In our improved version, we replaced the second boundary value problem by any Robin problem, and we require only the uniqueness of the Dirichlet problem for one suitable interval. Our proof is different from that in [2]. We formulate our result for second-order equations of the Emden-Fowler type, namely, those in which $u^{\prime}$ does not appear explicitly. There is no loss of generality since a change of the independent variable can transform more general equations into this type.

\section{Theorem 4. Suppose that the differential equation}

$$
u^{\prime \prime}(r)+f(r, u)=0
$$

is superlinear, namely, that $f(r, u) / u$ is a positive strictly increasing function of $u$ for each fixed $r$. Let $u(r)$ be a positive solution of (4.3) on a bounded interval $(a, b)$ satisfying the boundary conditions (4.1) and (4.2). Suppose that $u(r)$ can be extended to a solution of $(4.3)$ in a larger interval $\left(a_{1}, b_{1}\right) \supset(a, b)$ such that $u\left(a_{1}\right)=u\left(b_{1}\right)=0$, and it is known that this is the only positive solution of the Dirichlet problem on $\left(a_{1}, b_{1}\right)$. Then $u(r)$ is the unique solution of the original Robin boundary value problem on $(a, b)$.

Proof. Suppose that there is another solution $U(r)$. We first look at the case in which neither boundary condition is of the Dirichlet type. Assume that $U(a)>u(a)$. The case $U(a)<u(a)$ can be treated similarly. We extend $U(r)$ to be defined on $\left(a_{1}, b_{1}\right)$. By Lemma $1, U(r)$ must intersect $u(r)$ once inside $(a, b)$. We claim that they must intersect at least one more time at or before $b_{1}$, and before $U(r)$ changes sign. If they do not, then $u(r)>U(s)$ for all $r \in\left[b, b_{1}\right]$. This contradicts the second part of Lemma 1. Likewise $u(r)$ and $U(r)$ must intersect once more in $\left[a_{1}, a\right)$. In other words, $u(r)$ and $U(r)$ intersect at least three times in $\left[a_{1}, b_{1}\right]$. It is easy to see that the same is true even when one of the boundary conditions is of Dirichlet type. We next invoke the continuous deformation argument, already used once in the proof of Theorem 1, to construct a second positive solution to the Dirichlet problem on $\left(a_{1}, b_{1}\right)$, thus arriving at a contradiction. We keep track only of that portion of $U(r)$ between its first and third intersection points with $u(r)$.

We deform the solution $U(r)$ by pulling the first intersection point towards $a_{1}$, always making sure that $U(r)$ remains distinct from $u(r)$ (by keeping the value $\left|U^{\prime}(r)-u^{\prime}(r)\right|$ at the first intersection point away from 0$)$. Since the two solutions cannot be tangent to each other at any point and $U(r)$ cannot be tangent to the $r$-axis, the intersection points can vanish only through one of the endpoints. Either the first or the third intersection point will reach the (left 
or right, respectively) endpoint first. Without loss of generality we may assume that it is the first intersection point, and so now we have $U\left(a_{1}\right)=u\left(a_{1}\right)$.

Let us first consider the situation in which $U^{\prime}\left(a_{1}\right)>u^{\prime}\left(a_{1}\right)$. We now deform $U(r)$ by progressively increasing $U^{\prime}\left(a_{1}\right)$ towards $\infty$. We claim that if $U^{\prime}(a)$ is sufficiently large, either $U(r)>u(r)$ for all $r \in\left(a_{1}, b_{1}\right]$ or $U(r)$ crosses the $r$-axis somewhere in $\left(a_{1}, b_{1}\right)$ before intersecting $u(r)$ for a third time. In other words, the third intersection point must eventually disappear (and that can happen only through $b_{1}$ ). It then follows from continuity that there must be some intermediate choice of $U^{\prime}\left(a_{1}\right)$ for which the third intersection point is exactly at $b_{1}$, giving another solution to the Dirichlet problem on $\left(a_{1}, b_{1}\right)$. We give only a heuristic argument for verifying the claim. It is not hard to put it in a rigorous form. First of all, when all three intersection points are present, the second one cannot be too close to the right endpoint $b_{1}$, because oscillation of the difference $u(r)-U(r)$ cannot occur within too short a distance. Now if $U^{\prime}\left(a_{1}\right)$ is sufficiently large, then the maximum of $U(r)$ must be very large; otherwise $f(r, u)$ will remain uniformly bounded and therefore will not be large enough to change $U^{\prime}(x)$ from $U^{\prime}(a)$ to 0 at the maximum. If $U(r)$ is not larger than $u(r)$ for all $r$, then by the mean value theorem somewhere between the maximum of $U(r)$ and the second intersection point, the slope $U^{\prime}(r)$ must be very negative. It then follows that the slope $U^{\prime}(r)$ at the second intersection point must be very negative, so much so that $U(r)$ will not have enough room to bend upwards; it must therefore cross the $r$-axis within a very short distance form the second intersection point.

Let us now consider the situation in which $U^{\prime}\left(a_{1}\right)<u^{\prime}\left(a_{1}\right)$. We deform $U(r)$ by progressively decreasing $U^{\prime}\left(a_{1}\right)$. When $U^{\prime}\left(a_{1}\right)$ is very small, we see that the second intersection point will be very close to the right endpoint $b_{1}$. A simple Sturm comparison argument shows that $U(r)$ will not be able to intersect $u(r)$ again within the short distance before $b_{1}$. In other words, the second intersection point has disappeared. Thus there must be an intermediate choice of $U^{\prime}\left(a_{1}\right)$ for which the second intersection point is exactly at $b_{1}$, and we again have a contradiction.

We now have the following extension of Theorem 1:

Theorem 5. If in addition to the hypotheses of Theorem 1,

$$
g(a) \geq 0 \text { and } g(b) \geq 0,
$$

then equation (2.1) has at most one positive solution satisfying the Robin boundary conditions (4.1) and (4.2).

Proof. We enlarge the domain of $g(x)$ to $(-\infty, \infty)$ by defining $g(x)=g(a)$ for $x<a$, and $g(x)=g(b)$ for $x>b$. We can easily see that any solution of the boundary value problem can be extended to some larger interval $\left(a_{1}, b_{1}\right)$ as required in Theorem 4. The conclusion of the theorem then follows from Theorems 1 and 4.

Because of the presence of the first-order term in (3.8), extendability of a solution satisfying the Robin conditions may not be always guaranteed. We therefore include the extendability assumption for the general case in the formulation of the extension of Theorem 2 . 
Theorem 6. Let $n \geq 3$. Suppose that (3.6) holds on $(0, \infty)$ and that all solutions of the boundary value problem for the equation (3.8) with boundary conditions

$$
u^{\prime}(a)+A u(a)=0, \quad u^{\prime}(b)+B u(b)=0, \quad 0<a<b<\infty,
$$

can be extended to a solution of (3.8) on a larger interval $\left(a_{1}, b_{1}\right) \supset(a, b)$ with $u\left(a_{1}\right)=u\left(b_{1}\right)=0$. Then (3.8) has at most one solution satisfying (4.5).

$A$ special case where extendability of the solution holds is when $A \leq 0, p$ is subcritical, and $q(r) \geq 0$ is nondecreasing.

Proof. We need to establish the assertion only for the special case. If $a=0$, we are back to Theorem 2. If $a \geq 0$, then the fact $u^{\prime \prime}+m u^{\prime} / r<0$ implies that $u^{\prime}(r)<0$ for $r<a$. If $u(r)$ does not vanish between 0 and $a$, it must cut the $u$-axis with a positive slope, and it is easy to see that this contradicts the singularity of the first-order term. The extendability of $u(r)$ beyond $b$ is a well-known fact concerning the oscillation of the solutions of the Emden-Fowler equation (3.8).

Another example for which extendability can be established occurs when all solutions of the liner part of (3.8), namely, $U^{\prime \prime}+m U^{\prime} / r+q(r) U=0$, are oscillatory. It then follows from the Sturm comparison theorem that all solutions of (3.8) must be oscillatory.

An interesting comparison theorem can be derived from Theorem 6, according to the work in [13]. Suppose

$$
k_{1}(r) \geq 1 \text { and } k_{2}(r) \geq 1 \text { are nondecreasing on }[0, \infty) .
$$

Let $u_{1}(r)$ and $u_{2}(r)$ be positive solutions in an interval $(a, b)$ of the following equations, respectively:

$$
u_{1}^{\prime \prime}(r)+\frac{m}{r} u_{1}^{\prime}(r)+k_{1}(r) u_{1}^{p}+q(r) u_{1}=0,
$$

and

$$
u_{2}^{\prime \prime}(r)+\frac{m}{r} u_{2}^{\prime}(r)+k_{1}(r) u_{2}^{p}+k_{2}(r) q(r) u_{2}=0 .
$$

Let $u(r)$ be a solution of (3.8) in the same interval, and let $u(r)$ have a finite zero when extended beyond the right endpoint $b$. Furthermore, assume that all three solutions satisfy the same initial conditions

$$
u(a)=u_{1}(a)=u_{2}(a), \quad u^{\prime}(a)=u_{1}^{\prime}(a)=u_{2}^{\prime}(a) .
$$

Theorem 7. Suppose that (4.6) and (3.6) hold on $(0, \infty)$ and that $u(r), u_{1}(r)$, and $u_{2}(r)$ are solutions of (3.8), (4.7), and (4.8), respectively, such that (4.9) holds and $u(r)$ has a finite zero beyond $b$. Then

$$
u(x) \geq u_{1}(x), \quad x \in(a, b) .
$$

Furthermore, if $u^{\prime}(a) \leq 0$, then

$$
u(x) \geq u_{2}(x), \quad x \in(a, b) .
$$

Proof. We refer to [14] for the proof.

A situation in which the extendability hypothesis of Theorem 4 may not be automatically guaranteed by the form of the equations in Theorem 6 arises when 
the coefficient $g(x)$ of $(2.1)$ is negative. Comparison of the energy function at the endpoints is complicated by the fact that the term involving $g(x) u^{2}(x)$ no longer drops out at the boundary. We are able to obtain only a less satisfactory result. We can relax one but not both of the boundary conditions to that of the Robin type, and we need more assumptions on the functions $f(u)$ and $q(x)$. On the other hand, under these more restrictive requirements, we can replace the linear term in (2.1) with one having an exponent less than unity. More precisely, we consider equations of the form

$$
u^{\prime \prime}(x)+f(u(x))=G(x) u^{q}(x), \quad x \in(a, b), G(x) \geq 0,0 \leq q \leq 1,
$$

subject to the boundary conditions

$$
u(a)=0 \quad \text { and } \quad u^{\prime}(b)+B u(b)=0 .
$$

The case $q=0$ can be considered as an Emden-Fowler equation with a forcing term.

We require in addition to (2.4) that

$$
\frac{F(u)}{u^{q+1}} \text { is a strictly increasing function of } u
$$

and, in place of $(2.5)$, the stronger condition that

$$
G(x)=-g(x) \text { is nondecreasing in }(a, b) .
$$

Theorem 8. Under the assumptions (2.4), (4.14), and (4.15), the boundary value problem (4.12)-(4.13) has at most one positive solution.

Proof. Superlinearity holds by virtue of the sign of $G(x)$ and the value of $q$.

Suppose there are two distinct solutions, $u(x)$ and $U(x)$, to the boundary value problem.

Define $r(x)=u^{\prime}(x) / u(x)$ and $R(x)=U^{\prime}(x) / U(x)$ as in the proof of Theorem 1 . The second boundary condition in (4.13) becomes

$$
r(b)=R(b) \text {. }
$$

Let our solutions be named such that

$$
u^{\prime}(a)<U^{\prime}(a)
$$

By Lemma $1, r(x)>R(x)$ for $x$ in a small right neighborhood $(a, a+\varepsilon)$ of $a$. We may as well assume that the inequality holds throughout the entire interval $(a, b)$. Indeed, if there is a $\tau \in(a, b)$ for which $r(\tau)=R(\tau)$, we simply replace the original boundary value problem by one defined on the subinterval $(a, \tau)$. by

We proceed as in the proof of Theorem 1, replacing the definition of $E(u)$

$$
E(u)=\frac{u^{\prime 2}}{2}+F(u)-\frac{G u^{q+1}}{q+1}
$$

and noting that (2.8) and (2.9) hold with $c=b$. The two energy identities (2.13) and (2.14) become

$$
E(u(b))=u^{\prime 2}(a)-\int_{a}^{b} \frac{G^{\prime}(x) u^{q+1}(x) d x}{q+1}
$$




$$
\gamma^{q+1} E(U(b))=\gamma^{q+1} U^{\prime 2}(a)-\int_{a}^{b} \frac{G^{\prime}(x) \gamma^{q+1} U^{q+1}(x) d x}{q+1} .
$$

At $x=b$,

$$
u^{q+1}(b)=\gamma^{q+1} U^{q+1}(b)
$$

but

$$
u^{\prime 2}(b) \geq \gamma^{q+1} U^{\prime 2}(b) .
$$

By (4.14),

$$
\frac{F(u(b))}{u^{q+1}(b)} \geq \frac{F(U(b))}{U^{q+1}(b)} .
$$

It follows from this inequality and (4.21) that

$$
F(u(b)) \geq \gamma^{2} F(U(b)) .
$$

We thus see that

$$
E(u(b)) \geq \gamma^{2} E(U(b)) .
$$

At the other endpoint $a$, since $u(a) \leq U(a)$,

$$
u^{\prime 2}(a) \leq U^{\prime 2}(a)<\gamma^{q+1} U^{\prime}(a) .
$$

A contradiction is then obtained as in the proof of Theorem 1.

\section{The generalized Matukuma equation}

In this section we consider the equation

$$
\Delta u(x)+q(|x|) u^{p}(x)=0, \quad x \in \mathbb{R}^{n},
$$

where $n \geq 3,1<p<(n+2) /(n-2)$, and $q(r)>0$ is a $C^{1}$-function. In view of symmetry, we can turn to the equivalent ordinary differential equation

$$
u^{\prime \prime}(r)+\frac{n-1}{r} u^{\prime}(r)+q(r) u^{p}(r)=0, \quad u^{\prime}(0)=0, r>0 .
$$

There are three types of solution: (1) those with a finite zero, (2) those with a finite mass

$$
\int_{0}^{\infty} \frac{u^{p}(r) r^{n-1} d r}{1+r^{2}}<\infty
$$

and (3) those with an infinite mass. The existence of each type of solution and its dependence on the initial height $u(0)$ were studied in great detail by $\mathrm{Ni}$ and Yotustani [21]. Yanagida [24] gave an affirmative answer to the conjecture concerning the uniqueness of the solution with finite mass in the special case that $q(r)=1 /\left(1+r^{2}\right)$. His proof actually works without much change for more general coefficients.

The transformation

$$
\begin{gathered}
u(r)=v(s) r^{1-n / 2}, \\
r=e^{s},
\end{gathered}
$$


gives the new equation

$$
v^{\prime \prime}(s)-k^{2} v(s)+\left(e^{s}\right)^{\eta} q\left(e^{s}\right) v^{p}=0,
$$

where

$$
k=\frac{m-1}{2}
$$

and

$$
\eta=\frac{(n+2)-p(n-2)}{2}>0 .
$$

Following Yanagida, we take one of the solutions with finite mass and denote it by $\phi(s)$. The initial condition $u^{\prime}(0)=0$ and the finiteness of mass translate into

$$
\lim _{s \rightarrow \pm \infty} \phi(s)=0 \text {. }
$$

Now the problem is reduced to one that is "symmetrical" with respect to $-\infty$ and $\infty$, in the sense that the two "endpoints" are no longer distinguishable. The property of the coefficient that makes Yanagida's proof work is that the function

$$
Q(s)=\frac{2}{p+1}\left(e^{s}\right)^{\eta} q\left(e^{s}\right)
$$

satisfies the $\wedge$-condition. This is equivalent to the simpler condition that

$$
\left(r^{\eta} q(r)\right)^{\prime} \text { changes sign once for } r>0 \text {. }
$$

Let $\sigma$ be a point such that

$$
Q^{\prime}(s) \geq 0 \text { for } s<\sigma \text { and } Q^{\prime}(s) \leq 0 \text { for } s>\sigma .
$$

Note that $\sigma$ is not unique since $Q^{\prime}(s)$ may vanish in an entire interval.

The energy identity for equation (5.6) is

$$
v^{\prime 2}(s)-k^{2} v^{2}(s)+Q(s) v^{p+1}=\int_{-\infty}^{s} Q^{\prime}(t) v^{p+1}(t) d t .
$$

Assertion (ii) of Yanagida's Lemma 2.2 is equivalent to

$$
z(s)=\phi^{\prime}(s)+k \phi(s)>0 \text { for all } s .
$$

This can be verified directly by observing that (5.6) gives $z^{\prime}-k z=\phi^{\prime \prime}-k^{2} \phi<0$ and that $\lim _{s \rightarrow \infty} z(s)=0$. Upon reflection, we also have

$$
\phi^{\prime}(s)-k \phi(s)<0 \text { for all } s .
$$

This is, in fact, equivalent to the fact that $u^{\prime}(r)<0$.

Let $\psi(s)$ be another solution of finite mass. By virtue of superlinearity, we see that $\psi(s)$ must intersect $\phi(s)$ at least once. As in [24], we show that $\phi(s)$ and $\psi(s)$ cannot intersect for a second time. Suppose they do. From the intermediate value theorem, there must be a first point $s_{1}$, between the first and the second intersection points, at which $\phi^{\prime}\left(s_{1}\right) / \phi\left(s_{1}\right)=\psi^{\prime}\left(s_{1}\right) / \psi\left(s_{1}\right)$. We may assume that $s_{1} \leq \sigma$. The contrary case can be reduced to the present one by a reflection. Before the first intersection point, the two ratios $\phi^{\prime}(s) / \phi(s)$ and $\psi^{\prime}(s) / \psi(s)$ cannot coincide. Suppose the contrary and there exists a point 
$\tau<s_{1}$ at which $\phi^{\prime}(\tau) / \phi(\tau)=\psi^{\prime}(\tau) / \psi(\tau)$. We can take $\tau$ to be the smallest of all such points. Integrating $\phi^{\prime \prime}(s) \psi(s)-\phi(s) \psi^{\prime \prime}(s)$ over $(-\infty, \tau)$ gives $\phi^{\prime}(\tau) \psi(\tau)-\phi(\tau) \psi^{\prime}(\tau)=0$. On the other hand, using the differential equation to eliminate $\phi^{\prime \prime}(s)$ and $\psi^{\prime \prime}(s)$ shows that the expression $\phi^{\prime \prime}(s) \psi(s)-\phi(s) \psi^{\prime \prime}(s)$ is nonnegative and nontrivial in $(-\infty, \tau)$, an obvious contradiction. Let us assume that $\phi\left(s_{1}\right) \geq \psi\left(s_{1}\right)$. As in the proof in $\S 2$, we conclude that the ratio $\phi(s) / \psi(s)$ is a monotone function in $\left(-\infty, s_{1}\right)$ :

$$
\frac{\phi(s)}{\psi(s)} \leq \frac{\phi\left(s_{1}\right)}{\psi\left(s_{1}\right)}=\gamma .
$$

By assumption, $\gamma \geq 1$. Multiplying the energy identity satisfied by $\psi(s)$ with the constant $\gamma^{p+1}$, and then subtracting the identity satisfied by $\phi(s)$, we obtain

$$
\left(\gamma^{p-1}-1\right)\left(\phi^{\prime 2}(s)-\phi^{2}(s)\right) \geq 0 .
$$

This contradicts $(5.14)$ and (5.15).

Again we see that the ratios $\phi^{\prime}(s) / \phi(s)$ and $\psi^{\prime}(s)$ actually must be different for all $s \in(-\infty, \infty)$. Thus $\phi(s) / \psi(s)$ is a monotone function for all $s \in$ $(-\infty, \infty)$. Let

$$
\delta=\phi(\sigma) / \psi(\sigma)
$$

Extending (5.13) to the whole $s$-axis and using the fact that $\phi(s), \psi(s) \rightarrow 0$ as $s \rightarrow \infty$, we see that

$$
\int_{-\infty}^{\infty} Q^{\prime}(t) \phi^{p+1}(t) d t=0
$$

and

$$
\int_{-\infty}^{\infty} Q^{\prime}(t) \psi^{p+1}(t) d t=0
$$

Incidentally, (5.19) implies that if $G(t)$ is monotone, or equivalently if $G^{\prime}(t)$ does not change sign, then (5.1) does not have a finite mass solution. Subtract the first identity from $\delta^{p+1}$ times of the second one. We obtain a contradiction because by (5.12), the difference of the left-hand side of (5.19) and (5.20) is a strictly positive number.

We have thus proved an extension of Yanagida's result.

Theorem 9. Suppose that (5.11) holds. Then the differential equation (5.1) can have at most one positive solution with finite mass.

\section{The Matukuma equation-General boundary CONDitions}

A natural question to ask next is whether uniqueness still holds for other boundary value problems on the Matukuma equation. For example, is there only one solution that vanishes on the sphere of given radius $b$ and is positive in the interior of the ball? More generally, given $0<a<b<\infty$, we consider solutions of

$$
u^{\prime \prime}(r)+\frac{n-1}{r} u^{\prime}(r)+q(r) u^{p}(r)=0, \quad p>1
$$

such that

$$
u(a)=u(b)=0 \text { and } u(r)>0 \text { for } r \in(a, b)
$$


Results in this section do not require $p$ to be subcritical. If $a$ is chosen to be 0 , the singularity of the second term in (6.1) dictates that the only possible boundary condition at $a$ is of the Neumann type

$$
u^{\prime}(0)=0 \text {. }
$$

In this case, a restriction on the upper bound of $p$ (which may be different from the critical value $(n+2) /(n-2)$, depending on the actual form of $q(r))$ is perhaps needed, but only for ensuring the existence of a solution to the boundary value problem, rather than being required by the uniqueness proof.

Theorem 4 can be used to replace the Dirichlet condition with one of the Robin type, under suitable conditions.

Yanagida's technique does not appear to be extendable to general boundary conditions. The main obstacle is that (5.14) and (5.15) are no longer valid. The method also fails to include supercritical $p$.

Instead we turn to an alternative approach that Kwong and Zhang [15] recently applied to equations of the form $\Delta u+f(u)=0$. We shall derive several uniqueness criteria. The first is a stronger form of (5.11). We require that $(5.11)$ holds for all $\eta>0$, not just for the particular one given by (5.8). It is easy to see that an equivalent condition is

$$
\frac{r q^{\prime}(r)}{q(r)} \text { is nonincreasing in }(a, b) \text {. }
$$

This is reminiscent of the condition imposed on $f(u)$ in [15]. The method is actually applicable to include the case $b=\infty$, but we consider only finite $b$ here for the sake of simplicity.

The ideas will only be sketched since the method is similar to that of the result in [15]. The Kolodner-Coffman method reduces the proof of uniqueness to showing that given any solution $u(r)$ of the boundary value problem, the solution $w(r)$ of the linearized equation

$$
\begin{gathered}
L[w]=w^{\prime \prime}+\frac{n-1}{r} w^{\prime}+p q(r) u^{p-1} w=0, \\
w(a)=0, \quad w^{\prime}(a)=1,
\end{gathered}
$$

changes sign exactly once in $(a, b]$. By superlinearity, $w(r)$ must change sign at least once. What remains is to make sure that $w(r)$ does not change sign for a second time. When the interval is sufficiently short, this situation is known to be valid. For the simpler equation $\Delta u+f(u)=0$, this fact is implied by Theorem 1.7 of $\mathrm{Ni}$ and Nussbaum [20]. Lemma 12 in [2] confirms this assertion for general equations. A continuation argument then lets us extend this assertion to general $(a, b)$ provided we can show that under no circumstances can $w(r)$ have exactly one zero inside $(a, b)$ and $w(b)=0$. In [15], this crucial step is implemented by establishing Lemma 7 , which is stated below in a more general form.

Lemma 2. Suppose that $w(b)=0$ and that $w(r)$ has only one zero $\rho$ inside $(a, b)$. Let $z(r)$ be a $C^{2}$ function defined in $[a, b]$ such that

$$
\begin{aligned}
& L[z(r)] \leq 0 \quad \text { for } r \leq \rho, \\
& L[z(r)] \geq 0 \quad \text { for } r \geq \rho .
\end{aligned}
$$

If either $z(a)>0$ or $z^{\prime}(a)>0$, then $z(b)>0$. 
Proof. The proof makes use of Sturm's comparison theorem. We examine the oscillation of $z(r)$ as we move from the endpoint $a$ towards $b$. At the beginning, $L[z(r)]$ has the correct sign to ensure that $z(r)$ oscillates faster than $w(r)$; thus, $z(r)$ has a zero before $\rho$. Once passed $\rho, z(r)<0$; now the comparison theorem works in the reverse way, and $z(r)$ oscillates more slowly than $w(r)$. As a result, $z(r)$ cannot have another zero before $\rho$. But once passed $\rho, L[z]$ changes sign, and $z(r)$ changes pace. Hence $z(r)$ has a zero between the two zeros, $\rho$ and $b$, of $w(r)$. After this second zero of $z(r)$, the oscillation slows down again and $z(r)$ cannot have a zero before or at $b$. We thus conclude that $z(r)$ changes exactly twice in $[a, b]$, and therefore $z(b)>0$.

If we can construct a suitable function $z(r)$ satisfying (6.7), with $z(a)>0$ but $z(b)<0$, then Lemma 2 implies that $w(r)$ cannot have a zero at $b$, and the uniqueness proof is complete. In [15], the function $z=r u^{\prime}+\beta u$, with a suitable choice of the constant $\beta$ was used.

For the Matukuma equation, direct computation gives

$$
L\left[r u^{\prime}+\beta u\right]=\left[\beta(p-1)-2-\frac{r q^{\prime}(r)}{q(r)}\right] q(r) u^{p} .
$$

We now choose $\beta$ so that the expression inside the square brackets vanishes at $\rho$. By virtue of (6.4) the right-hand side of $(6.8)$ has exactly the required sign property (6.7), and $z(a)=a u^{\prime}(a)>0$, and $z(b)=b u^{\prime}(b)<0$. We have thus proved uniqueness under condition (6.4).

By experimenting with more general trial functions of the form $z=h(r) u^{\prime}+$ $k(r) u$, we have come up with two other useful ones for which the corresponding expression $L[z]$ is particularly simple:

$$
L\left[r^{m} u^{\prime}+\beta u\right]=\left[\beta(p-1)-2 m r^{m-1}-\frac{r^{m} q^{\prime}(r)}{q(r)}\right] q(r) u^{p}
$$

and

$$
\begin{aligned}
& L\left[r^{2-m} u^{\prime}+(m-1) r^{1-m} u+\beta u\right] \\
& \quad=\left[\beta(p-1)+(m p+m-p-3) r^{1-m}-\frac{r^{2-m} q^{\prime}(r)}{q(r)}\right] q(r) u^{p} .
\end{aligned}
$$

They give rise, respectively, to the criteria

$$
r^{m-1}\left(\frac{r q^{\prime}(r)}{q(r)}+2 m\right) \text { is nonincreasing in }(a, b)
$$

and

$$
\frac{1}{r^{m-1}}\left(\frac{r q^{\prime}(r)}{q(r)}-(m p+m-p-3)\right) \text { is nonincreasing in }(a, b) .
$$

A positive linear combination of the comparison functions

$$
z=\left(\lambda r+\mu r^{m}+\varphi r^{2-m}\right), \quad \lambda, \mu, \nu \geq 0,
$$

can also be used. 
Theorem 10. Let $0<a \leq b<\infty$. If the coefficient $q(r)$ satisfies any one of the conditions (6.4), (6.11), or (6.12) (or more generally if some positive linear combination of the expressions in these conditions is nonincreasing), then (6.1) has at most one solution satisfying (6.2). If $a=0$, the same conclusion is valid for the boundary condition (6.3).

Simple examples of functions that satisfy (6.4) include all powers (positive or otherwise) of $r$ and $1 /\left(1+r^{\kappa}\right)$ for all $\kappa$. Products of positive powers of functions that satisfy (6.4) also satisfy (6.4). It is also easy to see that if $q(r)$ satisfies (6.4), so does $q(1 / r)$.

Coefficients of the form

$$
q(r)=\alpha+\frac{1}{1+r^{2}}, \quad \alpha>0
$$

have been studied by Toland [23]. However, the condition (6.4) is not satisfied unless $b$ is small. Theorem 3 of $\S 3$ also fails. It is interesting to ask whether uniqueness is still valid for these equations.

Theorem 10 furnishes a complete description for the structure of the solutions of the Matukuma equation.

Theorem 11. Under the same hypotheses as Theorem 10, there exists a positive extended number $\mu$ that divides the solutions of (5.2) into three types:

1. If $u(0)=\mu$, the solution $\phi(r)$, which we call the ground state, is an entire (positive for all $r>0$ ) solution that decays with the fastest rate as $r \rightarrow \infty$.

2. If $u(0)>\mu$, the solution is an entire solution that decays with a rate slower than that of the ground state (the ratio $u(r) / \phi(r)$ goes to $\infty$ as $s \rightarrow \infty$ ).

3. If $u(0)<\mu$, the solution has a finite zero, which is a strictly decreasing function of $u(0)$.

In the case that $\mu=\infty$, the first two types are void.

To close the section, we point out a major difference between the result here and that obtained in [15], and we indicate some further extensions. What the proof exploits is the sign property, not the actual magnitude, of the right-hand side of (6.7). In the case of all the identities used in this section, the value of $u(r)$ at each $r$ plays no role. In [15], the right-hand side of the corresponding (6.7) has the required sign property only if we know that $u(r)$ is nonincreasing in the interval $[a, b]$. For this reason, the result there is established only when a Neumann condition is satisfied at the left endpoint $a$. On the other hand, the function $f(u)$ in [15] is allowed to assume negative values for $u$ small, and that monotonicity requirement on $u f^{\prime}(u) / f(u)$ is relaxed when $f(u)<0$.

In searching for other comparison functions for Lemma 2 , we have tried $z=h(r) u^{\prime}+k(r) u$, with any $h(r)$ and $k(r)=m h(r) / 2 r-h^{\prime}(r) / 2+\beta$. Direct computation gives

$$
\begin{aligned}
L[z]= & {\left[\beta(p-1)+\frac{m(p-1) h(r)}{2 r}-\frac{(p+3) h^{\prime}(r)}{2}-\frac{h(r) q^{\prime}(r)}{q(r)}\right] q(r) u^{p} } \\
& -\left[\frac{h^{\prime \prime \prime}(r)}{2}+\frac{m(m-2)\left(h(r)-r h^{\prime}(r)\right)}{2 r^{3}}\right] u .
\end{aligned}
$$

In fact, the test functions used above are the three linearly independent solutions of the differential equation obtained by equating the coefficients of $u$ in (6.15) to zero. It is interesting to see what uniqueness criterion can be obtained 
by choosing other $h(t)$. The existence of the second term in the right-hand side, however, means that, in general, uniqueness results obtained by using (6.15) apply only to Neumann-Dirichlet problems. Take a very simple example: $n=3$ and $h(t)=t^{3} . L[z]$ has the correct sign for a suitable choice of $\beta$ if

$$
q(r) \text { and } r^{2}\left(\frac{r q^{\prime}(r)}{q(r)}+\frac{p+11}{2}\right)
$$

are both increasing in $(a, b)$; hence the Neumann-Dirichlet problem has at most one solution. form

Another possible extension of the method is to equations of the more general

$$
\Delta u+f(|x|, u)=0 .
$$

In particular, uniqueness of the radially symmetry solution can be proved for Neumann-Dirichlet problems for

$$
\Delta u+q(|x|) u^{p}-u=0, \quad p>1,
$$

when $q(r)$ is nonincreasing and satisfies (6.4). The monotonicity of $q(r)$ is needed in two places. First it implies that $u(r)$ is a nonincreasing function in $(a, b)$. Then it gives the appropriate structure of the right-hand side of (6.7) so that $\beta$ can be chosen as desired to make the proof successful. We omit the straightforward details.

\section{FURTHER EXAMPLES AND EXTENSIONS}

Even though Theorem 1 is false in general for coefficients satisfying the $V$ property, uniquenss can still be proved in some specific situations. The types of result obtained in this section are less satisfactory and apply to coefficients with rather special structures. For this reason, we have refrained from formulating general propositions, other than Theorem 12, and merely indicate the ideas in some examples.

We first observe that a proof similar to that of Theorem 1 gives the following "uniqueness" result for fixed-end boundary value problems, which is of interest in itself.

Theorem 12. Let $u(x) \geq 0$ and $U(x)$ be two solutions of

$$
u^{\prime \prime}(x)+f(u(x))+g(x) u(x)=0, \quad x \in(a, b),
$$

in the interval $(a, b)$ with the assumptions on the initial values

$$
\begin{gathered}
u(a)=U(a)>0, \quad u^{\prime}(a)<0, \\
\left|U^{\prime}(a)\right|<\left|u^{\prime}(a)\right| \quad \text { or } \quad U^{\prime}(a)=-u^{\prime}(a) .
\end{gathered}
$$

If the coefficient $q(r)$ is nonnegative and the uniqueness criteria of Theorem 1 , $(2.4),(4.14)$, and $(2.5)$ are satisfied, then $u(x)$ and $U(x)$ cannot intersect again in $(a, b]$.

As a consequence, given any two fixed-end conditions

$$
u(a)=\alpha>0, \quad u(b)=\beta>0,
$$

there are at most two solutions to the boundary value problem. Suppose there are two distinct solutions, denoted by $u_{1}(x)$ and $u_{2}(x)$. At a (or b), the two 
initial slopes, $u_{1}^{\prime}(a)$ and $u_{2}^{\prime}(a)$, cannot both be negative; however, they can both be positive. In case $u_{1}^{\prime}(a)$ is negative, then

$$
\left|u_{1}^{\prime}(a)\right|<u_{2}^{\prime}(a) .
$$

Proof. At $a, r(a)=u^{\prime}(a) / u(a)<U^{\prime}(a) / U(a)=R(a)$. We need to show that $r(x) \neq R(x)$ for all $x>a$. This will imply the first conclusion of the theorem. Indeed, if $u(x)$ and $U(x)$ intersect before $b$, the intermediate value theorem will entail the existence of an interior point $\rho$ at which $r(\rho)=R(\rho)$. The case in which $u(x)$ and $U(x)$ intersect at $b$ right on the $r$-axis can be treated in the same way if the point $c$ in (3.6) is different from $b$. The remaining case is done by using a limiting argument as in the proof of Theorem 1.

Suppose that there is an interior point at which $r(x)=R(x)$. Take the smallest of such points. Since we wish to make use of the analogy with Theorem 1 and there is no danger of confusion, let us rename this point $b$. Then $u(x)<$ $U(x)$ for all $x \in(a, b]$; hence the ratio $\gamma=u(c) / U(c) \geq 1$. Following the proof of Theorem 1 , we consider the energy functions and derive a contradiction using (2.13) and (2.14). The initial condition (4.17) ensures that $E(u(a)) \leq$ $E(U(a)) \leq \gamma E(U(a))$. The rest of the proof runs in exactly the same way as that of Theorem 1.

Let us turn our attention to the fixed-end boundary value problem. It follows easily from the first conclusion of the theorem that not both $u_{1}^{\prime}(a)$ and $u_{2}^{\prime}(a)$ can be negative.

We claim that $u_{1}(x)$ and $u_{2}(x)$ cannot intersect other than at the endpoints. Suppose they do at some $\rho \in(a, b)$. By applying the first conclusion to the subinterval $(\rho, b)$, we see that the slopes $u_{1}^{\prime}(\rho)$ and $u_{2}^{\prime}(\rho)$ cannot both be negative. By applying the first conclusion now to the problem on $(a, \rho)$ (after doing a reflection), we see that the two slopes at $\rho$ cannot be both positive either. Suppose $u_{1}^{\prime}(\rho)<0<u_{2}^{\prime}(\rho)$. Using the first conclusion on the subinterval $(\rho, b)$, we see that we must have $\left|u_{1}^{\prime}(\rho)\right|<\left|u_{2}^{\prime}(\rho)\right|$. Considering the other subinterval gives exactly the opposite inequality, and thus we have a contradiction.

Now suppose there is a third solution $u_{3}(r)$. By what we proved in the last paragraph, none of the three solutions can intersect in the open interval $(a, b)$. Without loss of generality we may assume that $u_{1}(r)<u_{2}(r)<u_{3}(r)$, for all $r \in$ $(a, b)$. A standard Sturm comparison argument using the superlinearity of the equation show that $u_{3}(r)-u_{2}(r)$ must oscillate strictly faster than $u_{2}(r)-u_{1}(r)$, contradicting the fact that both functions have the same zeros in $[a, b]$.

This theorem has an obvious application in determining the structure of the solutions shot out from a fixed initial height at a fixed point $a$. We do not pursue the straightforward details. Instead we use Theorem 12 to study (3.8) with a symmetric and $\wedge \wedge$-shaped $q(r)$.

Example 1. Consider (3.8) on a symmetric interval $(-b, b)$, with a symmetric $q(r)$ :

$$
q(r)=q(-r) \text { and } q(r) \text { is } \wedge \text {-shaped in }(0, b) .
$$

In particular, if $q(r)$ is nondecreasing in $(0, b)$, then $q(r)$ has the $\vee$-property in $(-b, b)$. We show that the Dirichlet problem of $(3.8)$ on $(-b, b)$ has a unique solution which therefore must be symmetric with respect to the origin. 
Of course we still have, by Theorem 1 , uniqueness and symmetry of the solution if $q(r)$ is assumed to be nonincreasing in $(0, b)$; the symmetry conclusion agrees with a result of Gidas, $\mathrm{Ni}$, and Nirenberg in [10].

Suppose there is a nonsymmetric solution $u(x)$. By reflection, $u_{2}(x)=$ $u(-x)$ is a distinct solution that intersects $u(x)$ at $x=0$. Also $u^{\prime}(0)=u_{2}^{\prime}(0)$. Applying the last conclusion of Theorem 12 to $(0, b)$ gives a contradiction. Existence of a solution is well known.

Example 2. Take $(a, b)=(0,4)$. Choose $q(r)$ to be any function such that

$$
q(0)=1, q(1), q(4) \geq \frac{1}{9}, r^{2} q(r) \text { is nondecreasing in }(0,1)
$$

and

$$
q(r) \text { has the } \wedge \text {-property in }(1,3) .
$$

As an example we can choose $q(r)$ to be $(3-2 x) / 3$ in $(0,1)$ and any nondecreasing function in $(1,3)$; then $q(r)$ has the $V$-property. We can still establish uniqueness.

We first claim that the maximum of a solution $u(x)$ must occur in $[1,4)$. Suppose the contrary and $u(x)$ attains its maximum at $\rho<1$. By scaling (compressing horizontally) the part of $u(x)$ in $(\rho, 4)$, and reflecting, we obtain a function that fits into $(0, \rho)$ and satisfies a differential equation similar to (3.8) but with a larger factor in the reaction term. We can now compare this function with the original $u(x)$ in $(0, \rho)$. The scaling factor is so large that we can easily conclude that the scaled function must oscillate faster than $u(x)$, but this contradicts the fact that both functions require the same distance $\rho$ from their respective maximum to vanish.

Next we make use of the Kolodner-Coffman method. According to the method, we shall have uniqueness if we can show that corresponding to any solution $u(x)$ of the Dirichlet problem, a solution $w(r)$ of the first variational equation

$$
w^{\prime \prime}(r)+\left[p u^{p-1}(r)+q(r)\right] w(r)=0
$$

cannot change sign more than once in $(a, b)$. By the Sturm separation theorem, we need only look at the particular solution $w(r)$ such that $w(\rho)=0$. The required property of $w(r)$ can be proved by examining the oscillatory behavior of $w(r)$ in each of the subintervals $(0, \rho)$ and $(\rho, b)$. By Theorem 1 of [14], the monotonicity of $r^{2} q(r)$ in $(0, \rho)$ implies that $w(r)$ cannot change sign in $(0, \rho)$. On the other hand, Theorem 12 implies that $w(r)$ cannot change sign in $(\rho, b)$. Hence the only change of sign occurs at $\rho$, and uniqueness of the Dirichlet problem is established.

Example 3. The equation

$$
u^{\prime \prime}(x)+x^{4}\left(u^{5}+u\right)=0
$$

has been shown in [14] to have the uniqueness property for Dirichlet problems. The change of variables

$$
u(x)=\frac{v(t)}{\sqrt{x}}, \quad t=x^{2},
$$

transforms the equation into

$$
v^{\prime \prime}(t)+\frac{v^{5}(t)}{4}+\left(\frac{3}{16 t^{2}}+\frac{t}{4}\right) v(t)=0
$$


The coefficient of $v(t)$ satisfies the $\vee$-property but is positive for all $s$. Contrast this with the counterexample in $\S 3$ for which the coefficient changes sign.

\section{REFERENCES}

1. F. V. Atkinson and L. A. Peletier, Emden-Fowler equations involving critical exponents, Nonlinear Anal. 10 (1986), 755-766.

2. C. Bandle and Man Kam Kwong, Semilinear elliptic problems in annular domains, Z. Angew. Math. Phys. 40 (1989), 245-257.

3. H. Brezis and L. Nirenberg, Positive solutions of nonlinear elliptic equations involving critical Sobolev exponents, Comm. Pure Appl. Math. 36 (1983), 437-477.

4. C. Budd and J. Norbury, Semilinear elliptic equations and supercritical growth, J. Differential Equations 68 (1987), 169-197.

5. Chiun-Chuan Chen and Chang-Shou Lin, Uniqueness of the ground state solution of $\Delta u+$ $f(u)=0$ in $R^{n}, n \geq 3$, Comm. Partial Differential Equations 16 (1991), 1549-1572.

6. C. V. Coffman, On the positive solutions of boundary value problems for a class of nonlinear differential equations, J. Differential Equations 3 (1967), 92-111.

7. C. V. Coffman, and M. Marcus, Existence and uniqueness results for semilinear Dirichlet problems in annuli, Arch. Rational Mech. Anal. 108 (1989), 293-307.

8. E. N. Dancer, The effect of domain shape on the number of positive solutions of certain nonlinear equations, J. Differential Equations 74 (1988), 120-156.

9. B. Gidas, W. M. Ni, and L. Nirenberg, Symmetry and related properties via the maximum principle, Comm. Math. Phys. 68 (1979), 209-243.

10. __ Symmetry of positive solutions of nonlinear elliptic equations in $R^{n}$, Adv. in Math. Studies 7A (1981), 369-402.

11. Man Kam Kwong, On the Kolodner-Coffman method for the uniqueness problem of EmdenFowler BVP, Z. Angew. Math. Phys. 41 (1990), 79-104.

12. __ Uniqueness of positive solutions of $\Delta u-u+u^{p}=0$ in $R^{n}$, Arch. Rational Mech. Anal. 105 (1989), 243-266.

13. _ A comparison result and elliptic equations with subcritical growth, Argonne National Laboratory Preprint Series MCS-P73-0589 (1989); to appear in Proc. of Conference on Reaction Diffusion Equations, Gregynog, Wales, 1989.

14. __ Uniqueness results for Emden-Fowler boundary value problems, Nonlinear Anal., Theory Methods \& Appl. 16 (1991), 435-454.

15. Man Kam Kwong and L. Zhang, Uniqueness of the positive solution of $\Delta u+f(u)=0$ in an annulus, Differential Integral Equations 4 (1991), 583-599.

16. Yi $\mathrm{Li}$ and W. M. Ni, On conformal scalar curvature equations in $R^{n}$, Duke Math. J. 57 (1988), 895-924.

17. __ On the existence and symmetry properties of finite total mass solutions of Matukuma equation, Eddington equation and their generalizations, Arch. Rational Mech. Anal. 108 (1989), 175-194.

18. T. Matukuma, The cosmos, Iwanami Shoten, Tokyo, 1938. (Japanese)

19. K. McLeod and J. Serrin, Uniqueness of positive radial solutions of $\Delta u+f(u)=0$ in $R^{n}$, Arch. Rational Mech. Anal. 99 (1987), 115-145.

20. W. M. Ni and R. Nussbaum, Uniqueness and nonuniqueness for positive radial solutions of $\Delta u+f(u, r)=0$, Comm. Pure Appl. Math. 38 (1985), 69-108.

21. W. M. Ni and S. Yotsutani, Semilinear elliptic equations of Matukuma-type and related topics, Japan J. Appl. Math. 5 (1988), 1-32.

22. E. S. Noussair and C. A. Swanson, Solutions of Matukuma's equation with finite total mass, Indiana Univ. Math. J. 38 (1989), 557-561. 
23. J. F. Toland, Positive solutions of nonlinear elliptic equations-existence and nonexistence of solutions with radial symmetry in $L_{p}\left(R^{N}\right)$, Trans. Amer. Math. Soc. 282 (1984), 335-354.

24. E. Yanagida, Structure of positive radial solutions of Matukuma's equation, Preprint.

25. Liqun Zhang, Private communication.

Mathematics and Computer Science Division, Argonne National laboratory, ARgONNE, IlLINOIS 60439

E-mail address: kwong@mcs.anl.gov

Department of Mathematics, University of Rochester, Rochester, New York 14620

E-mail address: lyii@uhura.cc.rochester.edu 